\title{
Correlation Between Pyridoxal 5'-Phospate Level And Valproic Acid In Epilepsy Children
}

\author{
I Gusti Ngurah Made Suwarba, Ni Putu Yunik Novayanti, I Gusti Lanang Sidiartha, \\ Dewi Sutriani Mahalini \\ ${ }^{1}$ Child Health Department, Faculty of Medicine, Udayana University/Sanglah General Hospital, Bali, Indonesia
}

Background: It is important to maintain the adequate level of vitamin B6 to ensure stable metabolism. Vitamin B6 serum level might decreased by absorption disturbance or increasing demand. Valproic acid increase the synthesis of serum GABAergic in the other hand vitamin B6 is required as cofactor for gamma-amino butyric acid (GABA) formation. The dosage and duration of valproic acid therapy might be correlated with vitamin B6 serum level. The aim of this study is to know the correlation between vitamin B6 serum level against dosage and duration of valproic acid therapy in children with epilepsy.

Materials and Methods: This is a cross sectional study to investigate the correlation between vitamin B6 serum level against dosage and duration of valproic acid therapy. The level of vitamin B6 serum was determined by checking vitamin B6 active form in serum, pyridoxal 5'-phospate (PLP).

Results: In this study, 37 epilepsy children with valproic acid duration therapy more than 3 months was enrolled. Fifty six percent epilepsy children were male, commonly on children age 1-5 years old. Spearman correlation coefficient test showed a significant weak negative correlation between vitamin $\mathrm{B} 6$ serum level and dosage of valproic acid $(\mathrm{r}=-0.35 ; p=0.03)$, and very weak negative correlation with valproic acid duration therapy $(\mathrm{r}=-0.08 ; p=0.59)$, however it was not significant.

Conclusion: There was a significant weak negative correlation between vitamin B6 serum level and very weak negative correlation with valproic acid duration therapy, but not significant in children with epilepsy.

Keywords: correlation, valproic acid, vitamin B6

\section{Introduction}

There is some role of Vitamin B6 in various metabolisms of multiple enzymes which is important to maintain the adequate level of vitamin B6 serum. The abundant of availability food-rich vitamin B6 in developing countries make the incident of deficiency of vitamin B6 due to low intake is less frequent. ${ }^{1}$ However, certain conditions can decrease the level of vitamin B6.

Many literatures has reported about the low level of vitamin B6 in the usage of anti-epileptic drugs.,3,4 Antiepileptic drugs (AED) is thought to reduce the level of

Date of submission: October 10, 2018

Last Revised: December 10, 2018

Accepted for publication: December 11, 2018

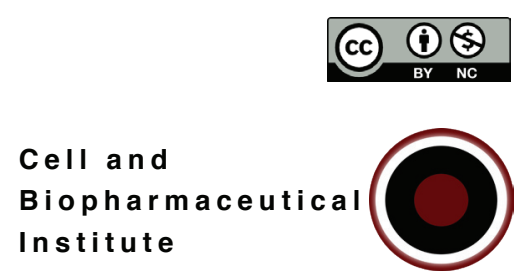

Corresponding Author:

I Gusti Ngurah Made Suwarba

Child Health Department

Faculty of Medicine, Udayana University/Sanglah General Hospital

J. P.B. Sudirman, Denpasar, Bali, Indonesia

E-mail: suwarbangurah@yahoo.co.id 
vitamin B6 serum, presumably because most of antiepileptic drugs act as direct enzyme inducers which can modulate the activity of enzymes in liver. ${ }^{4}$ Induction metabolism of those enzymes can cause depletion of involving cofactor such as vitamin B6, folic acid and vitamin B12. ${ }^{4}$ The liver enzymes reaction which involved the vitamin B6 (pyridoxal 5 '-phosphate) are aspartate aminotransferase and alanine aminotransferase.

The level of vitamin B6 serum can be determined quantitatively using levels of pyridoxal 5-phosphate (PLP) as one of the active vitamin B6 (vitamer) form which is circulating in the blood. PLP level can be detected in plasma, serum and cerebrospinal fluid. Previous studies showed that the mean PLP plasma level of epileptic children decreased significantly after 20 weeks of anticonvulsant administration. ${ }^{3}$ More than $50 \%$ of epileptic children treated with both valproic acid and carbamazepine were found with plasma PLP levels $<30 \mathrm{nmol} / \mathrm{L}$ (below the normal value limit). ${ }^{2}$ Sener, et al., has found that the mean level of vitamin B6 was lower in the AED group than in non-AED group. ${ }^{4}$ Valproic acid is one of the most widely used AED in current management of epilepsy in children because it is the first choice agent for most types of epilepsy, both symptomatic and idiopathic. ${ }^{5}$ Unlike other types of AED, valproic acid is not inducer enzyme. ${ }^{5}$ However valproic acid works by increasing the synthesis of GABAergic in which vitamin B6 acts as cofactor in the formation of am-aminobutyric acid (GABA). The increment synthesis of GABA, increases the demand of vitamin B6 and may affect the level of vitamin B6 in blood. In this study we want to know whether valproic acid correlates with the level of vitamin B6 in serum. In order to know it, this study was conducted to determine the correlation between vitamin B6 against dosage and the duration of valproic acid usage.

\section{Materials and methods}

The design of this study was cross-sectional, conducted from June 2017 to January 2018. This is the first study which was correlated PLP levels with doses of valproic acid in the particular population of children. The current fact is AED an enzyme inducer which can decreases the PLP levels. However, in the other hand valproate acid is not an enzyme inducer. The study sample was epileptic children whom treated in Sanglah Hospital and received valproic acid therapy more than 3 months, due to the average AED therapy dose can be achieved within 3 months of the initial therapy. Clinical indication of vitamin B6 examination is that if there is a downward trend it is necessary to consider vitamin B6 supplementation when administering valproic acid.

This is a correlation study, this result cannot be implemented yet, however, it has been able to raise the issue that vitamin B6 not only decreases due to AED which are an enzyme inducers, but also by non-inducer enzymes. Based on the vitamin B6 insert kit we used, the results of this examination cannot be used to diagnose but only for research, so the vitamin B6 levels that we get cannot be categorized as low, normal, or high. However the data on vitamin B6 levels will be useful if we do a comparison/ relationship analysis.

Exclusion criteria included epileptic children with severe infections, Crohn's disease, consume INH, malnutrition, impaired kidney function and liver function, had vitamin B6 supplementation in the last 2 months. Sample was collected consecutively.

Nutritional status was determined when the patient became a sample by measuring body length in $\mathrm{cm}$ and weighing body weight (BW) expressed in $\mathrm{kg}$, ideal $\mathrm{BW}$ determined based on body lenght plotted on the CDC curve for ages $>5$ years and WHO curve for age $\leq 5$ years. Nutritional status is determined based on the actual percentage of $\mathrm{BW} /$ ideal $\mathrm{BW}$ which is categorized as obese $>120 \%$, overweight $>110-120 \%$, well nourish $90-110 \%$, malnutrition $<90 \%$.

The minimum sample size is calculated based on the calculation of single sample size with correlation coefficient was set at $0.410 .{ }^{4}$ The determination $\alpha 0.05$ and $\beta$ is set at $80 \%$, so the minimum sample size was 20 .

Data including identity, age, gender, body weight, height, and clinical variables (such as onset of epilepsy, history of febrile seizures or epilepsy in family, type of epilepsy, the dosage of valproic acid, duration of valproic acid) were recorded from eligible samples. Epilepsy onset is the age at the first time diagnosed with epilepsy as noted in the medical record, expressed in months. History of epilepsy and/or febrile seizures in the family, stated "Yes" if in one generation level (first degree relatives) the family has a history of epilepsy or febrile seizures in the family, otherwise stated "No". The duration of administration of valproic acid is the time period when the patient begins to receive epilepsy with valproic acid (monotherapy) until it 
is sampled, expressed in months. The type of seizure is a general or focal type of seizure according to the diagnosis in the medical record or that has been adjusted to the EEG results. The dose of valproic acid is the total dose of valproic acid in milligrams consumed by the patient in a day divided by the body weight of the patient when used as a case expressed in $\mathrm{mg} / \mathrm{kgBW} /$ day.

After recording, $3 \mathrm{~mL}$ of blood was drawn by laboratory officer. Blood samples were taken with a clear red lid tube with no carbon-covered anticoagulant (plain tube), centrifuged as soon as the sample was received to get the serum. Serum stored in opaque plastic none translucent and stored in frozen conditions at a temperature of $-40^{\circ} \mathrm{C}$. Serum storage in our study follows the procedure applicable in the Sanglah Hospital clinical pathology laboratory.

In principle, the serum must be stored at a temperature of no more than $-20^{\circ} \mathrm{C}$, PLP is stable in the refrigator for up to 5 years at $-70^{\circ} \mathrm{C}$. After all samples were collected, vitamin B6 was examined at once. Vitamin B6 serum level was expressed by the level of PLP in unit of picomol/liter. Examination techniques using enzymelinked immunosorbent assay (ELISA) using reagents from BioAssay Technology Laboratory-Human Vitamin B6 ELISA Kit. Elisa sandwich method is cheaper than high performance liquid chromatography (HPLC).

Statistical analysis was performed using computer (SPSS.20). The data distribution was analyzed by ShapiroWilk test and categorized as normal distribution if $p$-value more than 0.05. Correlation test for PLP level, drug dosage and duration of valproic acid were analyzed by Spearman test. Informed consent was made to the parents of subjects who met the inclusion and exclusion criteria. Blood collection and data were carried out after informed consent was signed. This research has been approved by Research Ethics Commission, Faculty of Medicine, Udayana University with protocol study number: 1389/UN14.2.2/ $\mathrm{PD} / \mathrm{KEP} / 2018$.

\section{Results}

This study involved 37 epileptic children whom were treated with valproic acid. Normality test by Shapiro-Wilk on PLP level, dosage of valproic acid and duration of valproic acid usage showed that the data were not distributed normally, while data of age were distributed normally. Onset of epilepsy, nutritional status, and duration of treatment are displayed in ordinal variables. Table 1 shows the characteristics of subjects.

The mean age of this study was 5.5 years, and 22 $(59.5 \%)$ was male. The onset of epilepsy mostly (57\%) started at the age of 1-5 years. Twenty-seven percent of subjects had history of febrile seizures and or epilepsy in family. Approximately $59.5 \%$ of epilepsy subjects were well-nourished, the remaining $22 \%$ were undernourished, $13.5 \%$ obese, and $5.4 \%$ more nutrition. The distribution of PLP levels in this research vary widely with middle value was $325 \mathrm{pmol} / \mathrm{L}$ and ranged from 67.9 to $1902.6 \mathrm{pmol} / \mathrm{L}$. Four from five obese subjects had PLP level below the median $(<325 \mathrm{pmol} / \mathrm{L})$, meanwhile 1 obese subject with PLP value $325 \mathrm{pmol} / \mathrm{L}$. While the remaining had various value. Types of epilepsy based on seizure type and etiology showed $59.5 \%$ were idiopathic epilepsy and $86.5 \%$ with general epilepsy manifestation.

The three variables (PLP level, valproic acid dosage, duration of valproic acid) that would be tested by the hypothesis were numerical variables with non normal distribution.

Based on the Spearman correlation test, there was a significant weak negative correlation between PLP serum level and dosage of valproic acid, and there was a very weak negative correlation between PLP serum level and duration of valproic acid usage but the correlation was not significant (Table 2).

\section{Discussion}

Vitamin B6 is a water soluble vitamin which is found in many foods. Vitamin B6 involves in metabolism of proteins, carbohydrate and fat in body. The involvement of vitamin B6 in the reaction is closely related to the function of the nervous system, immune system and endocrine. ${ }^{2,6}$ PLP is one of the active forms of vitamin B6 which acts as cofactor in glutamate acid decarboxylase reaction by changing glutamate into GABA. ${ }^{7,8}$ Thus, considering the vital role of vitamin B6 in body metabolism, deficiency of vitamin B6 could affect body metabolism reaction. Valproic acid as the most widely used antiepileptic drug today was showed by several studies can decrease vitamin B6 level in subjects whom use valproic acid.

Our research subjects were epilepsy patients with average of 5.5 years old. Fifty-nine percent of them were male. This incidence was more frequent in males, in accordance with previous study, in which $56.9 \%$ of epileptic 
Table 1. Characteristics of of epileptic children whom received valproic acid.

\begin{tabular}{|c|c|c|}
\hline \multirow{2}{*}{ Study characteristics } & \multicolumn{2}{|c|}{$(n=37)$} \\
\hline & Unit & Value \\
\hline Gender, male & $\mathrm{n}(\%)$ & $22(59.5)$ \\
\hline Onset of epilepsy, months old & median (minimum-maximum) & $24(1-110)$ \\
\hline Age, months old & mean (SD) & $65.9(37.3)$ \\
\hline Has family history of febrile seizure or epilepsy & $\mathrm{n}(\%)$ & $10(27)$ \\
\hline \multicolumn{3}{|l|}{ Nutritional status } \\
\hline Obese, & $\mathrm{n}(\%)$ & $5(13.5)$ \\
\hline Overweight & $\mathrm{n}(\%)$ & $2(5.4)$ \\
\hline Well nourished & $\mathrm{n}(\%)$ & $22(59.5)$ \\
\hline Malnutrition & $\mathrm{n}(\%)$ & $8(22)$ \\
\hline PLP, pmol/L & median (minimum-maximum) & $325(67.9-1902.6)$ \\
\hline Valproic acid dosage, $\mathrm{mg} / \mathrm{kg} /$ day & median (minimum-maximum) & $20(15-40)$ \\
\hline \multicolumn{3}{|l|}{ Duration of Valproic acid, months } \\
\hline$<20$ months & $\mathrm{n}(\%)$ & $23(62.2)$ \\
\hline$\geq 20$ months & $\mathrm{n}(\%)$ & $14(37.8)$ \\
\hline Epilepsy type general seizure & $\mathrm{n}(\%)$ & $32(86.5)$ \\
\hline Idiopathic epilepsy & $\mathrm{n}(\%)$ & $22(59.5)$ \\
\hline
\end{tabular}

pmol/L: picomol per liter; mg/kg/day: milligram/kilogram/day; SD: standard deviation.

patients were males. ${ }^{9}$ Onset of epilepsy commonly started in ages 1 to 5 years old. This result was different from previous study which showed epilepsy onset below 1 year old..$^{9}$ This is likely due to the different inclusion and exclusion criteria of this study. Subjects with history of febrile seizures and epilepsy in our study was $27 \%$. Previous studies found $10 \%$ of patients with history of febrile seizures in family, and $13 \%$ with family history of epilepsy. ${ }^{9}$ A study in Turkey also found similar result, there was history of first degree relative $(16 \%)$, second degree relative $(6 \%)$, and third degree relative $(2.5 \%) .{ }^{10}$ Similar to previous studies, the subjects' nutritional status of this study were dominated by well nourish $57.5 \%$, followed by malnutrition $22 \%$ and obese $13.5 \%$. Previous studies found $69.9 \%$ well nourish, $21 \%$ undernourished, $6 \%$ obese. ${ }^{9}$ General epilepsy was found in $85.4 \%$ and $58.5 \%$ was idiopathic epilepsy. Similar to previous study $80.4 \%$ had general seizures, idiopathic epilepsy $74.5 \%{ }^{9}$

PLP level in serum or plasma is generally used to assess vitamin B6 status. Vitamin B6 level was said to sufficient at level of $20 \mathrm{nmol} / \mathrm{L}$, and sub-optimal when plasma PLP level are $10 \mathrm{nmol} / \mathrm{L} .{ }^{11}$ However, many literatures and studies have different cut off points, which is categorized to be normal if PLP level in serum or plasma $>30 \mathrm{nmol} / \mathrm{L}$, and deficiency if the level is below $20 \mathrm{nmol} / \mathrm{L} \cdot .^{2-4,7,10,12}$ Based on the recommended dietary allowance (RDA) to maintain adequate level of vitamin B6 in body, daily intake of vitamin B6 1.5-2 mg is highly required. ${ }^{13}$ Vitamin B6 has low toxicity, neuropathy symptoms will only appear in a dosage $1000 \mathrm{mg} /$ day of vitamin B6. ${ }^{14}$

Meassurement of vitamin B6 level can be done by several methods, such as spectrometry, chromatography, electrochemistry and enzymatic assay. ${ }^{15}$ Nowadays the diagnostic test using a HPLC method which result PLP level in international units $\mathrm{nmol} / \mathrm{L}$. In this study, researchers used sandwich ELISA as one of the enzymatic assay methods. This method is able to detect vitamin B6 in both serum and plasma with sensitivity up to $2.51 \mathrm{pmol} / \mathrm{L}$ (standard curve range 5-1500 pmol/L). PLP level from the results cannot be interpreted into deficiency or normal status. 
Table 2. Spearman correlation test results.

\begin{tabular}{lcc}
\hline & \multicolumn{2}{l}{ PLP level } \\
\hline Valproic acid dosage & $\mathrm{r}$ & -0.35 \\
& $p$ & 0.03 \\
Duration of valproic acid & $\mathrm{r}$ & -0.08 \\
& $p$ & 0.59 \\
\hline
\end{tabular}

In this study, the PLP level of subjects ranged from 67.9 to $1902.6 \mathrm{pmol} / \mathrm{L}$ with a median level of $325 \mathrm{pmol} / \mathrm{L}$. It was interesting finding in the distribution of PLP level from 4 out of 5 obese subjects whose PLP level below the median $(<325 \mathrm{pmol} / \mathrm{L}), 1$ obese subject with PLP values 325 $\mathrm{pmol} / \mathrm{L}$. While the other nutritional status were found vary. This is probably related to the chronic inflammation process that occurs in obese patients. However, this condition was not discussed in this study.

Previous study found that the average PLP plasma concentration decreased below normal values $(>30 \mathrm{nmol} / \mathrm{L})$ in $70 \%$ of patients with phenytoin, and more than $50 \%$ of patients with both valproic acid and carbamazepin and $22 \%$ of patients with lamotrigine, but it did not showed meaningful differences between those 4 groups. ${ }^{2}$ In several correlation studies there was no significant correlation between the duration of valproic acid therapy with PLP level. ${ }^{4,14}$ The median PLP value in this study was 325 $\mathrm{pmol} / \mathrm{L}$. Eighteen subjects (48.6\%) were obtained with PLP levels $<325 \mathrm{pmol} / \mathrm{L}$ where $61 \%$ were experienced by subjects with as valproate dosage $\geq 25 \mathrm{mg} / \mathrm{kg} / \mathrm{day}$. We do not know yet what dose of valproic acid is.

The results showed a significant negative correlation between vitamin B6 levels and the dose of valproic acid, the higher the dose the lower the vitamin B6. These results indicate a tendency to decrease due to valproate, a clinical implication considering the administration of vitamin B6 in epilepsy patients who get valproic acid. Further research is needed with different designs to prove the relationship between the two variables. The fact that there is currently is OAE enzyme inducer decreases PLP levels. But valproate acid is not an enzyme inducer. There have been no studies that have correlated PLP levels with doses of valproic acid in the particular population of children. In another study, it was stated that $50 \%$ of epilepsy patients experienced a decrease in PLP but not specifically mentioned the percentage decrease in users of valproate acid. Only $50 \%$ was mentioned in patients with val and carbamazepine, and no correlation was conducted in the study. ${ }^{2}$

Our study found a weak significant negative correlation, $\mathrm{r}=-0.35 ; p=0.03$, although it was weak in correlation, this result indicate the possibility of the higher dosage of valproic acid, the lower the plasma PLP level. Previous research by Sener, et al., stated that the mean of vitamin B6 level in the AED group was lower than non-AED group, but there was no significant difference in the average PLP in the two groups. Significant difference was found only in patients using carbamazepin which showed decrease tendency. ${ }^{4}$ This result was differ from our study's result, probably caused by different hypothesis test. Correlation study by Davis, et al., found 37\% epilepsy children had vitamin B6 level below the lowest limit of reference range and did not find relationship between decreased level of vitamin and anticonvulsants both monotherapy and polytherapy. ${ }^{11}$ These results were contradiction with our results. AED in the study of Davis, et al., was more diverse. In addition, there was difference of age between previous studies and this study, which the age of subjects in previous study was older than 10 years old. ${ }^{11}$ However, in this study, the mean age of subjects was 5.5 years old.

Valproic acid works by enhancing the GABAergic system by increasing GABA synthesis. ${ }^{16}$ This condition make a possibility that the higher the dose, the higher demand for vitamin B6 will be needed, which will decrease the level of PLP. Other drugs that have similar effect to valproic acid are benzodiazepine, tiagabine, vigabatrin, gabapentin, but all four drugs are GABAergic via different mechanism. Benzodiazepine works by increasing the function of GABA receptors, tiagabine and vigabatrin decreases GABA degradation and GABA reuptake, gabapentin increases GABA release through unclear mechanisms, although it is thought it can increase GABA synthesis, but some literature consistently stated only valproic acid which works by increasing GABA synthesis. ${ }^{16}$

This study found that $80.5 \%$ subjects have long duration of valproic acid usage more than 24 months. In the Spearman test, there was no correlation between vitamin B6 level and the duration of valproic acid usage $(\mathrm{r}=-0.08 ; p=0.59)$. Similar to previous studies that found no correlation between the duration of anti-epileptic drugs with vitamin B6, homocysteine, and vitamin B12. ${ }^{4}$ Similarly with previous study, there was no correlation between the duration of valproic acid therapy. 
The best measurement of vitamin B6 level can be obtained by combining other biomarkers such as: alkaline phosphatase (ALP), low albumin serum level, renal function and inorganic phosphate. The weakness of this study is we did not combine the vitamin B6 level with other inflammatory markers due to limited funding.

\section{Conclusion}

There is a significant weak correlation between vitamin B6 level with dosage of vaproat acid and weak negative correlation that was not significant between vitamin B6 level and the duration of valproic acid usage.

\section{References}

1. Ueland PM, Ulvik A, Avila LR, Midttun Q, Gregory JF. Direct and functional biomarkers of vitamin B6 status. Annu Rev Nutr. 2015; 35: 33-70.

2. Tamura T, Aiso K, Johnston KE, Black L, Faught E. Homocysteine, folate, vitamin B-12 and vitamin B-6 in patients receiving antiepileptic drug monotherapy. Epilepsy Res. 2000; 40: 7-15.

3. Attilakos A, Papakonstantinou E, Schulpis K, Voudris K, Katsarou E, Mastroyianni S, et al. Early effect of sodium valproat and carbamazepine monotherapy on homocysteine metabolism in children with epilepsy. Epilepsy Res. 2006; 71: 229-32.

4. Sener U, Zorlu Y, Karaguzel O, Ozdamar O, Coker I, Topbas M. Effect of common anti-epileptic drug monotherapy on serum levels of homocysteine, vitamin B12, folic acid and vitamin B6. Seizure. 2005; 15(2): 79-85.
5. Perucca E. Pharmalogical and therapeutic properties of valproat. A summary after 35 years of clinical experience. CNS Drugs. 2002; 16(10): 695-714.

6. Sweetman SC, editors. Martindale: The Complete Drug Reference. 35th ed. London: Pharmaceutical Press; 2007.

7. Dave HN, Ramsay RE, Khan F, Sabharwal V, Irland M. Piridoxine deficiency in adult patients with status epilepticus. Epilepsy Behav. 2015; 52(Pt A): 154-8.

8. Merril AH, Henderson M. Disease associated with defects in vitamin B6 metabolite, or utilization. Ann Re Nutr. 1987; 7: 137-56.

9. Suwarba IGN. Insiden dan karakteristik klinis epilepsi pada anak. Sari Pediatri. 2011; 13(2): 123-8.

10. Davis RE, Reed A, Smith BK. Serum pyridoxal, folate and vitamin B12 levels in institutionalized epileptics. Epilepsia. 1975; 16: 4638.

11. Leklem J. Vitamin B6: a status report. J Nutr. 1990; 120 Suppl 11(4): 1503-7.

12. WHO and FAO. Vitamin B6. In: Vitamin and Mineral Requirements in Human Nutrition. 2nd edition. Bangkok: World Health Organization and Food and Agriculture Organization of the United Nations; 2004. p.175-9.

13. Institute of Medicine. Vitamin B6. In: Dietary Reference Intakes for Thiamin, Riboflavin, Niacin, Vitamin B6, Folate, Vitamin B12, Pantothenic Acid, Biotin, and Choline. Washington DC: National Academy Press; 1998. p.150-95.

14. Vilaseca MA, Monrós E, Artuch R, Colomé C, Farré C, Valls C, et al. Anti-epileptic drug treatment in children: hyperhomocysteinaemia, B-vitamins and the $677 \mathrm{C}$, T mutation of methylenetetrahydrofolate reductase gene. Eur J Paediatr Neurol. 2000; 4(6): 269-77.

15. Ahmad I, Mirza T, Qadeer K, Nazim U, Vaid FHM. Vitamin B6: Deficiency diseases and methods of analysis. Pak J Pharm Sci. 2013; 26(5): 1057-69.

16. Greenfield LJ. Molecular mechanisms of antiseizure drug activity at GABAA reseptors. Seizure. 2013; 22(8): 589-600. 